\title{
Televisión, audiencias y debates electorales: hacia la multipantalla
}

\section{Television, audiences, and electoral debates: Towards multiscreen viewing}

\author{
José Rúas-Araújo; Natalia Quintas-Froufe
}

Cómo citar este artículo:

Rúas-Araújo, José; Quintas-Froufe, Natalia (2020). "Televisión, audiencias y debates electorales: hacia la multipantalla". El profesional de la información, v. 29, n. 2, e290201.

https://doi.org/10.3145/epi.2020.mar.01

Artículo invitado recibido el 27-02-2020

Aceptación definitiva: 07-03-2020

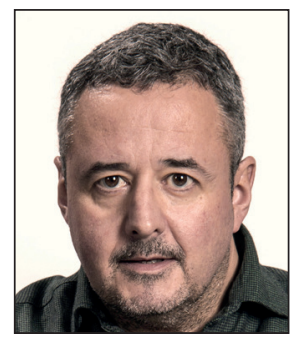

\author{
José Rúas-Araújo \\ https://orcid.org/0000-0002-1922-9644 \\ Universidad de Vigo, Facultad de Ciencias \\ Sociales y de la Comunicación \\ Campus Universitario da Xunqueira, s/n. \\ 36005 Pontevedra, España \\ joseruas@uvigo.es
}

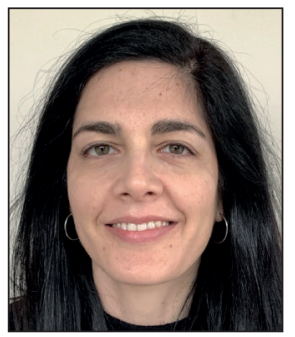

\author{
Natalia Quintas-Froufe \\ https://orcid.org/0000-0001-7597-6516 \\ Universidad de A Coruña, Facultad de \\ Ciencias de la Comunicación \\ Campus de Elviña. \\ 15007 A Coruña, España \\ n.quintas.froufe@udc.es
}

\section{Resumen}

En el actual ecosistema mediático, uno de los principales pilares de las campañas de comunicación de los partidos políticos se centra en la aparición de los líderes políticos en televisión. La presencia y exposición de los candidatos políticos en este medio, a través de diferentes formatos informativos, de entretenimiento o de infoentretenimiento, suele atraer la atención de gran parte de la ciudadanía, más allá de la duración de la propia campaña electoral. Los debates electorales son uno de los hegemónicos formatos televisivos de carácter político que reúnen a un mayor número de espectadores debido a las características particulares de los mismos. El interés de la audiencia por este tipo de formato, sin tradición en el contexto español, ha sido notable durante los veintiséis años de retransmisiones. La evolución de la audiencia de estos programas confirma el alto grado de atención que acaparan por parte de los espectadores españoles, al alcanzar históricas cuotas de pantalla en las cadenas que los emitieron, uno de los indicadores cuantitativos unánimemente aceptados en el estudio sobre audiencias. Sin embargo, la incorporación de nuevas pantallas y canales a los debates electorales ha ampliado el tradicional escenario en el que solían desarrollarse. La actual experiencia televisiva multipantalla y la participación de la audiencia a través de plataformas como las redes sociales suponen un reto para las estrategias de comunicación de los partidos políticos y los canales de televisión, al igual que un desafío para la medición de este nuevo público, significativamente más activo y generador de contenidos.

\section{Palabras clave}

Televisión; Audiencias; Debates electorales; Comunicación política; Multipantalla; Redes sociales; Twitter; Partidos políticos; Infoentretenimiento; Espectadores.

\footnotetext{
Abstract

In the current media ecosystem, one of the main pillars of political party communication campaigns is focused on the appearance of political leaders on television. The presence and exposure of political candidates in this medium, across different informative formats, from entertainment to infotainment, draws the attention of a considerable section of citizens beyond the electoral campaign. The electoral debates are one of the most hegemonic television formats relating

\section{Financiación}

Este trabajo forma parte del proyecto de investigación "DEBATv, Debates electorales televisados en España: modelos, proceso, diagnóstico y propuesta” (CSO2017-83159-R), proyecto de I+D+i (Retos) financiado por el Ministerio de Ciencia, Innovación y Universidades y por la Agencia Estatal de Investigación (AEI) del Gobierno de España, con el apoyo del Fondo Europeo de Desarrollo Regional (Feder) de la Unión Europea (UE).
} 
to politics, that brings together a large number of spectators. The interest of the Spanish audience in this type of format, without a historical tradition in Spain, has been remarkable to watch during the last twenty-six years of broadcasting. The evolution of the audience for these programs confirms a high level of interest by Spanish spectators and has reached historically high ratings for the networks broadcasting them, which is one of the quantitative indicators unanimously accepted as a standard in the study on audiences. However, the incorporation of new screens and channels into the electoral debates has widened the mainstream viewing scenario. The current multiscreen television experience and audience participation across platforms, such as social networks, represent a challenge for the communication strategies of political parties and television networks, as well as for measurement of this new audience, which is significantly more active, while also generating more content.

\section{Keywords}

Television; Audiences; Electoral debates; Political communication; Multiscreen; Social networks; Twitter; Political parties; Infotainment; Viewers.

\section{Introducción}

La televisión continúa siendo el medio más consumido y con mayor penetración en España (AIMC, 2020), y el medio de comunicación preferente para obtener información política (Humanes, 2014), de ahí que los principales partidos políticos decidan enviar sus mensajes a través de un medio que mantiene una posición hegemónica de cara a la audiencia española. La política en televisión atrae audiencias como ningún otro medio, es el soporte fundamental de la información política y consigue introducir en los hogares de los ciudadanos, con escaso tiempo, las imágenes de los aspirantes en la carrera política (Berrocal-Gonzalo, 2005).

Desde el último tercio del siglo XX, la televisión se convirtió en el espacio central de la confrontación electoral (LópezLópez; Oñate, 2019). Como ya anticipaba Martínez-Pandiani (2006, p. 72), sin televisión no hay debate y sin debate no hay política. Una situación que se ha acentuado en los últimos años, ya que se ha incrementado el papel de la televisión como

"vehículo privilegiado de escenificación de alternativas políticas, conflictos, estrategias y debates de toda clase" (López-García et al., 2018, p. 778).

En el marco de los programas televisivos políticos cobra especial relevancia el formato de los debates electorales televisados. El debate, como programa televisivo, constituye

"un reto al anacronismo de la audiencia cautiva" (Proaño, 2002),

ya que las audiencias son sujetos colectivos activos, capaces de integrar en su lógica social la relación con la televisión (Callejo-Gallego, 1995). El interés de la audiencia televisiva en los debates electorales queda reflejado en las históricas cuotas de pantalla que alcanzan, uno de los indicadores unánimemente aceptados en el estudio de la audiencia (Webster; Phalen; Lichty, 2014; Huertas-Bailén, 2002; Berrocal-Gonzalo, 2005).

Desde los primeros debates americanos, el debate televisado se ha posicionado como uno de los eventos más atractivos de campaña política en las elecciones generales o presidenciales de cualquier país (Téllez; Muñiz; Ramírez, 2010, p. 252), es decir, los eventos estrella de las campañas (García-Martín, 2015) o incluso el epítome de las mismas (Blais; Perrella, 2008). Se trata de un formato sin gran tradición en el contexto español pero capaz de aglutinar a audiencias masivas desde sus primeras retransmisiones, que se ha convertido en una oportunidad mediática de la que pueden extraer abundantes réditos todos los participantes (Cantavella et al., 2008).

Los efectos que el visionado de los debates puede tener sobre la audiencia han sido ampliamente investigados internacionalmente (Blais; Perrella, 2008; Benoit; Hansen; Verser, 2003; Yawn et al., 1998; Chaffee, 1978).

El marco teórico parte de teorías de la recepción (Lull, 1997; Morley, 1996), de la participación de la audiencia (Livingstone, 2013; Carpentier, 2001) y de la audiencia social (Selva, 2016; D’heer; Verdegem, 2015; Claes; Deltell, 2015); así como de las teorías sobre modelos y sistemas de relación entre los medios de comunicación y la política (Hallin; Mancini, 2004), convergencia (Jenkins, 2008), hibridación mediática (Chadwick, 2013) y de la función social de la televisión como servicio de interés general y público (Tremblay, 2016; Trappel, 2016; Marzal-Felici; Zallo-Elguezabal, 2016; Horowitz, 2015).

Además, también hay que destacar la necesidad de contribuir al desarrollo de un modelo de medios de comunicación públicos y democráticos (Horowitz, 2015), dotándolos de recursos necesarios para innovar y renovarse, generando una conversación con la audiencia que facilite la independencia, la universalidad y el servicio al interés público a través de una política mediática activa (Trappel, 2016), fortaleciendo la relación entre emisor y receptor (Bruun, 2016), que responda a las mutaciones tecnológico-sociales y a la formación de comunidades virtuales (Marzal-Felici; Zallo-Elguezabal, 2016).

El debate televisado se ha posicionado como uno de los eventos más atractivos de campaña política en las elecciones generales o presidenciales de cualquier país 


\section{Debates electorales televisados en el contexto español}

En la década de los setenta comenzó la verdadera expansión de los debates electorales, convirtiéndose en parte de la modernización y personalización de las campañas electorales en el mundo (Reinemann; Wilke, 2007). Sin embargo, en España habría que esperar hasta la década de los noventa para su inclusión en las estrategias políticas de los partidos. La tradición de los debates electorales en España se remonta al mes de mayo de 1993, cuando se retransmitieron los dos primeros debates televisados entre José María Aznar y Felipe González. La audiencia estaba expectante ante la novedad de este formato y ello se plasmó en las elevadas cifras de audiencia que lograron. El primero de ellos, emitido por Antena 3, consiguió una cuota de pantalla de 61,8\% (9.625.000 espectadores). El segundo, emitido por Telecinco, incrementó el número de espectadores (10.526.000) y, consecuentemente, la cuota de pantalla $(75,3 \%)$. Ambas cifras fueron sobresalientes y motivaron que ambos programas fueran uno de los espacios más vistos de toda la historia de los dos canales, exceptuando la programación deportiva.

Tras quince años sin debates electorales, la convocatoria de elecciones generales del año 2008 supuso su regreso a la programación televisiva. La Academia de las Ciencias y las Artes de Televisión programó dos debates entre los aspirantes a la Presidencia del Gobierno: José Luis Rodríguez Zapatero y Mariano Rajoy. Ambos debates fueron retransmitidos en simulcast en cadenas nacionales, autonómicas y locales. El primero de los debates, emitido el 25 de febrero de 2008, fue seguido mayoritariamente por la audiencia en $\operatorname{La} 1$ (36,4\% y 8.036 .000 espectadores) aunque si se suman las cuotas de pantalla de todas las cadenas en las que se retransmitió se alcanzó la cifra de 13.043 .000 espectadores $(59,1 \%)$ (Barlovento, 2019). Estos datos le supondrían ser el debate más visto de la historia de la televisión en España.

El segundo de los debates no consiguió alcanzar dichas cifras (11.952.000 espectadores y 56,3\% de cuota) aunque La 1 continuó siendo la cadena preferida por la audiencia para seguir el debate (32,6\% y 6.958 .000 espectadores). Las elevadas cifras de audiencia que lograron obtener los dos debates electorales los convirtieron en los programas políticos con mayor audiencia de los anteriores quince años (Gallego-Reguera; Bernárdez-Rodal, 2017).

Tres años más tarde se optó por celebrar únicamente un cara a cara televisado entre Alfredo Pérez Rubalcaba y Mariano Rajoy.

Tabla 1. Debates televisados, España, 1993-2019

\begin{tabular}{|c|c|c|c|c|c|}
\hline Fecha & Políticos & Debate & Cadena & Cuota \% & Espectadores \\
\hline \multirow{2}{*}{$\begin{array}{l}1993 \\
\text { Mayo }\end{array}$} & \multirow{2}{*}{$\begin{array}{l}\text { - José María Aznar (PP) } \\
\text { - Felipe González (PSOE) }\end{array}$} & $1^{\circ}$ & Antena 3 & 61,8 & 9.625 .000 \\
\hline & & $2^{\circ}$ & Telecinco & 75,3 & 10.526 .000 \\
\hline \multirow{4}{*}{$\begin{array}{l}2008 \\
\text { Febrero }\end{array}$} & \multirow{4}{*}{$\begin{array}{l}\text { - José Luis Rodríguez Zapatero (PSOE) } \\
\text { - Mariano Rajoy }(P P)\end{array}$} & \multirow{2}{*}{$1{ }^{\circ}$} & simulcast & 59,1 & 13.043 .000 \\
\hline & & & \multicolumn{3}{|c|}{ (La 1 obtuvo $36,4 \%$ y 8.036 .000 espectadores) } \\
\hline & & \multirow{2}{*}{$2^{\circ}$} & simulcast & 56,3 & 11.952 .000 \\
\hline & & & (La 1 obtuvo un 3 & 8.000 espe & \\
\hline \multirow{2}{*}{$\begin{array}{l}2011 \\
\text { Noviembre }\end{array}$} & \multirow{2}{*}{$\begin{array}{l}\text { - Alfredo Pérez Rubalcaba (PSOE) } \\
\text { - Mariano Rajoy }(P P)\end{array}$} & \multirow{2}{*}{ Único } & simulcast & 54,2 & 12.005 .000 \\
\hline & & & \multicolumn{3}{|c|}{ (La 1 obtuvo una cuota del $24,8 \%$ ) } \\
\hline \multirow{3}{*}{$\begin{array}{l}2015 \\
\text { Diciembre }\end{array}$} & $\begin{array}{l}\text { - Soraya Sáenz de Santamaría (PP) } \\
\text { - Pedro Sánchez (PSOE) } \\
\text { - Pablo Iglesias (Podemos) } \\
\text { - Albert Rivera (CS) }\end{array}$ & $1^{\circ}$ & $\begin{array}{l}\text { Antena } 3 \\
\text { La sexta }\end{array}$ & 48,2 & 9.233 .000 \\
\hline & \multirow{2}{*}{$\begin{array}{l}\text { - Mariano Rajoy (PP) } \\
\text { - Pedro Sánchez (PSOE) }\end{array}$} & \multirow{2}{*}{$2^{\circ}$} & simulcast & 48,7 & 9.728 .000 \\
\hline & & & \multicolumn{3}{|c|}{ (La sexta obtuvo $14,6 \%$ y 2.914 .000 espectadores) } \\
\hline \multirow[b]{2}{*}{$\begin{array}{l}2016 \\
\text { Junio }\end{array}$} & \multirow{2}{*}{$\begin{array}{l}\text { - Mariano Rajoy (PP) } \\
\text { - Pedro Sánchez (PSOE) } \\
\text { - Pablo Iglesias (Podemos) } \\
\text { - Albert Rivera (CS) }\end{array}$} & \multirow[b]{2}{*}{ Único } & simulcast & 57,0 & 10.496 .000 \\
\hline & & & \multicolumn{3}{|c|}{ (La sexta obtuvo $14,1 \%$ y 2.602 .000 espectadores) } \\
\hline \multirow{4}{*}{$\begin{array}{l}2019 \\
\text { Abril }\end{array}$} & \multirow{4}{*}{$\begin{array}{l}\text { - Pedro Sánchez (PSOE) } \\
\text { - Pablo Casado (PP) } \\
\text { - Albert Rivera (CS) } \\
\text { - Pablo Iglesias (Unidas Podemos) }\end{array}$} & \multirow{2}{*}{$1^{\circ}$} & RTVE & 43,0 & 8.886 .000 \\
\hline & & & \multicolumn{3}{|c|}{ (La 1 obtuvo $35,7 \%$ y 7.245 .000 espectadores) } \\
\hline & & 20 & Atresmedia & 48,7 & 9.477 .000 \\
\hline & & 2 & \multicolumn{3}{|c|}{ (Antena 3 obtuvo un $27,2 \%$ y 5.283 .000 espectadores) } \\
\hline \multirow{2}{*}{$\begin{array}{l}2019 \\
\text { Noviembre }\end{array}$} & \multirow{2}{*}{$\begin{array}{l}\text { - Pedro Sánchez (PSOE) } \\
\text { - Pablo Casado (PP) } \\
\text { - Albert Rivera (CS) } \\
\text { - Pablo Iglesias (Unidas Podemos) } \\
\text { - Santiago Abascal (Vox) }\end{array}$} & \multirow{2}{*}{ Único } & simulcast & 52,7 & 8.632 .000 \\
\hline & & & \multicolumn{3}{|c|}{ (La 1 obtuvo $20,8 \%$ de la cuota y 3.402 .000 espectadores) } \\
\hline
\end{tabular}

El 7 de noviembre de 2011 tuvo lugar el segundo debate más visto en España (12.005.000 espectadores de media y $54,2 \%$ de cuota de pantalla). El debate fue retransmitido en simulcast por diecisiete cadenas de televisión, siendo de nuevo La 1 la elegida mayoritariamente (24,8\%). 
Con la convocatoria de elecciones generales del año 2015, y el fin del bipartidismo en la política española, la Academia de la Televisión decidió organizar dos debates en el mes de diciembre. En el primero de ellos se incorporó a los líderes de las nuevas formaciones políticas y en otro se mantuvo el tradicional cara a cara. En el primero intervinieron Soraya Sáenz de Santamaría (Partido Popular), Pedro Sánchez (PSOE), Pablo Iglesias (Podemos) y Albert Rivera (Ciudadanos). La emisión del debate por Antena 3 y La sexta supuso agrupar a 9.233 .000 espectadores (48,2\% de cuota de pantalla).

La relevancia de este debate en el contexto político y audiovisual es fundamental ya que es el primer debate electoral en España en el que intervienen más de dos fuerzas políticas. España se sumó a la tendencia internacional, exceptuando Estados Unidos, de la incorporación de más participantes en los debates, tal como Anstead (2016) confirmó tras el estudio de los debates electorales en cuatro democracias parlamentarias (Australia, Alemania, Canadá y Reino Unido).

El segundo debate, el tradicional cara a cara entre Mariano Rajoy y Pedro Sánchez, fue emitido por doce cadenas y concentró a 9.728 .000 espectadores (48,7\% de cuota de pantalla). En este caso sería La sexta el canal elegido preferentemente por la audiencia (14,6\% de cuota y 2.914 .000 espectadores) y no La 1.

Solo seis meses más tarde, la nueva convocatoria electoral de 2016, motivó la organización de un nuevo debate electoral con los cuatro líderes políticos en el mes de junio. El debate, celebrado el 31 de junio, fue emitido en simulcast por diecisiete cadenas y fue seguido por 10.496 .000 espectadores ( $57 \%$ de cuota). La Sexta fue la cadena favorita de la audiencia para el seguimiento del debate $(14,1 \%$ y 2.602 .000 espectadores).

La doble cita electoral del año 2019 dio lugar a la celebración de tres debates electorales, algo inédito en el panorama televisivo español. Los dos primeros, realizados en días consecutivos, fueron organizados por RTVE y Atresmedia. El 22 de abril se emitió el primero de ellos, aglutinando a 8.886 .000 espectadores (43\% de cuota) siendo La 1 (con una audiencia media de 7.245 .000 espectadores y $35,7 \%$ de cuota de pantalla) el canal preferido por la audiencia entre los once que lo emitieron. Este debate fue la emisión no deportiva más vista de todo el año 2019. Al día siguiente, los candidatos debatieron en Atresmedia incrementando las cifras conseguidas el día anterior $(9.477 .000$ espectadores y $48,7 \%$ de cuota de pantalla) siendo Antena 3 el canal líder (5.283.000 espectadores y el 27,2\% de cuota). Los dos debates electorales se situaron entre los programas más vistos del año.

Siete meses más tarde, la inminente cita electoral tras la imposibilidad de la formación de Gobierno forzó la realización de un nuevo debate electoral en el que se incorporó por primera vez la fuerza política Vox. El primer debate con cinco candidatos, emitido el 4 de noviembre, no consiguió mejorar los resultados de audiencia alcanzados en abril (8.632.000 espectadores y $52,7 \%$ de cuota de pantalla) pero La 1 volvió a ser el canal líder (3.402.000 millones de espectadores y una cuota media de pantalla de $20,8 \%$ ).

Este breve estudio longitudinal de la audiencia televisiva de los debates electorales organizados con motivo de las convocatorias de elecciones generales en España confirma el alto grado de atención que la audiencia española muestra en el seguimiento de estos eventos mediáticos. Es un formato que parece no haberse desgastado con el tiempo, ya que el primer debate del año pasado consiguió ser, como se apuntaba con anterioridad, el programa más visto del año por la audiencia española. Aun así, parece que la escasa tradición de los debates electorales en España, comparada con otros países como EUA, no está reñida con el interés de la audiencia española en el visionado de los mismos.

Durante los 26 años analizados, la cadena pública parecía erigirse como el canal por el que tradicionalmente la audiencia prefería visionar estos espacios, sin embargo, cadenas como La sexta han forjado una sólida identidad ligada sobre todo a los espacios de información política.
La escasa tradición de los debates electorales en España, comparada con otros países como EUA, no está reñida con el interés de la audiencia española en su visionado

Obviamente, el contexto en el que se han desarrollado

estos debates ha cambiado profundamente y ha experimentado grandes transformaciones. No solo a nivel político-social con la irrupción de nuevos partidos políticos y la desafección de la política por parte de los ciudadanos, sino también en el plano estrictamente televisivo. La multiplicación de la oferta televisiva derivó también en la diversificación de formatos televisivos en los que presentar a los candidatos políticos que incluyen fórmulas de infoentretenimiento (López-García et al., 2018), que han derivado en un proceso de espectacularización de la política. En este tipo de espacios calificados en la categoría de politainment se suele exponer la cara más humana y amable del candidato, que se muestra más relajado al no estar sometido a la rigidez de tiempos y formato de los debates electorales.

La presencia de los candidatos en estos programas ha proliferado y se han incorporado como una cita ineludible de la agenda mediática del candidato. Aunque el origen del infoentretenimiento político en España se remonta a la década de los noventa (Las noticias del Guiñol o Caiga quien caiga) (Martín-Jiménez; Berrocal-Gonzalo, 2017), los actores políticos acuden a una amplia tipología de formatos que van desde programas matinales (Reguero-Sanz; Martín-Jiménez, 2010), tertulias políticas (Montagut; Carrillo, 2017), o programas de entretenimiento politizado (Vázquez-Barrio; Cebrián-Guinovart, 2017).

En 2015 se produjo el salto de los políticos a este tipo de espacios de entretenimiento, siendo sus apariciones una constante (López-Rico; Peris-Blanes, 2017). Un ejemplo de ello fue la participación de Albert Rivera y de Pablo Iglesias en el programa Salvados, que consiguió reunir a 5.214 .000 espectadores (25,2\% cuota de pantalla) y que fuera el séptimo 
programa más visto de 2015. Sin lograr superar las grandes audiencias de los debates, estos programas acaparan cifras destacables de espectadores por el interés de observar cómo interactúa un candidato político en un entorno diferente al habitual.
La multiplicación de la oferta televisiva derivó también en la diversificación de formatos televisivos en los que presentar a los candidatos políticos

\section{Televisión, redes sociales y política: integración de otras pantallas en el visionado}

En el ecosistema mediático actual la emisión del debate no se ciñe estrictamente a la retransmisión lineal por televisión. Otros medios y canales consiguen expandirlo a nuevas audiencias, como la audiencia social, a través de las redes sociales de los partidos políticos o de los propios canales. En el caso de los debates electorales existe una amplia bibliografía sobre el rol de las redes sociales antes, durante y después de los debates electorales televisados (Chadwick; O'Loughlin; Vaccari, 2017; Lago-Vázquez et al., 2016; Vergeer; Franses, 2016; Trilling, 2015; D’heer; Verdegem, 2015; Elmer, 2013; Deller, 2011).

Las redes sociales han permitido el acceso a la esfera pública de todo aquello que solo existía en el ámbito privado y han provocado el surgimiento de una audiencia social creadora de nuevos discursos sobre determinados temas (Echegaray-Eizaguirre; Peñafiel-Saiz, 2013). Estamos ante usuarios que tienen capacidad de producir contenidos políticos en función de aspectos sociológicos, como su educación o estatus social (Casero-Ripollés, 2017), y que acceden a la información a través de segundas pantallas por lo que su comportamiento es diferente al de las audiencias tradicionales.

La bidireccionalidad puede ser un factor relevante que alienta el debate entre los ciudadanos y los políticos y, en este sentido, las redes sociales contribuyen a generar una retroalimentación con los protagonistas de los debates electorales televisados, haciendo que los comentarios ya no sean exclusivos de los intervinientes y los periodistas, y facilitando la opinión de los ciudadanos del debate minuto a minuto, con lo que los protagonistas están obligados a dar lo mejor de sí en cada intervención (Barrientos; Caldevilla-Domínguez; Vargas-Delgado, 2019).

La bidireccionalidad puede ser un factor relevante que alienta el debate entre los ciudadanos y los políticos

De hecho, la existencia de plataformas de redes sociales que permiten los comentarios públicos en tiempo real como Twitter, facilitan la valoración de los momentos decisivos de los debates y actuaciones políticas (Campos-Domínguez, 2017).

Y si las encuestas de opinión pueden describirse como una "foto instantánea" de la realidad, el seguimiento y monitoreo de redes sociales constituyen -siguiendo con la metáfora- un "vídeo" que captura la opinión pública y las conductas en movimiento, distinguiendo entre tendencias instaladas, hechos coyunturales y temas emergentes (González-López, 2019).

Uno de los últimos estudios del Pew Research Center (2019) muestra que tres cuartas partes de los estadounidenses prefieren ver las noticias a través de la televisión e internet, más que leerlas a través de la prensa o escucharlas en la radio. Y aunque la televisión sigue siendo el medio de comunicación preferido por el $44 \%$ de los encuestados (frente al $46 \%$ registrado en 2016), las preferencias de búsqueda de información online pasaron del $28 \%$ registrado en 2016 al $34 \%$ de 2018, es decir, con una diferencia del $6 \%$ más, frente al $2 \%$ menos de la televisión.

Este estudio señala que, aunque los jóvenes de entre 18 y 29 años son los principales consumidores de redes sociales, la diferencia en el consumo de redes de esta generación con su predecesora (30-49 años) se acorta cada vez más.

En la encuesta de este instituto de opinión estadounidense también se destaca que, en la actualidad, siete de cada diez estadounidenses usan las redes sociales para conectarse entre sí, interactuar con el contenido de las noticias, compartir información y entretenerse. Para muchos usuarios norteamericanos, las redes sociales son parte de su rutina diaria: aproximadamente tres cuartos de los usuarios de Facebook y alrededor de seis de cada diez usuarios de Instagram visitan estos sitios al menos una vez al día.

Las últimas elecciones presidenciales celebradas en EUA muestran también que la mitad de los estadounidenses aseguran que se informaron sobre las últimas elecciones presidenciales a través de las redes sociales (Greenwood; Perrin; Duggan, 2016).

Por su parte, el Estudio anual de redes sociales de IAB (2019) muestra que el 85\% de los internautas de entre 16 y 65 años usan las redes sociales, lo que equivale a un total de 25,5 millones de personas en España. Este mismo estudio señala que las redes sociales son una fuente de información para el $55 \%$ de los usuarios y que el $31 \%$ de ellos siguen las cuentas de medios de comunicación y partidos políticos a través de las mismas, siendo el teléfono móvil (95\%), ordenador (92\%) y tablet (54\%), los dispositivos de consulta.

En cuanto a la frecuencia de uso de redes sociales, Whatsapp se sitúa en primer lugar, con un $97 \%$ de los usuarios que indican que lo consultan a diario (el $85 \%$ de ellos varias veces al día), seguido de Facebook, con un $73 \%$ de usuarios que reconocen consultarlo a diario (47\% de ellos varias veces al día) e Instagram, con un $70 \%$ de consulta diaria (46\% varias veces al día). Por su parte, Twitter confirma una tendencia a la baja, con un $53 \%$ de los usuarios que aseguran consultarlo a diario, 31\% de ellos varias veces al día. 
Este informe corrobora también la tendencia, observada ya en su estudio de 2018, de que la denominada Generación Z (usuarios de 16 a 23 años), utiliza un mayor número de redes sociales a la vez y se combina más dispositivos que la generación anterior, de los millennials (24 a 38 años), con una deriva cada vez más acentuada hacia el consumo de contenidos e información más rápida y menos permanente y hacia la denominada social TV, a través de vídeo y streaming.

Asimismo, el último de los informes y encuestas de Navegantes en la Red de la AIMC (2019), señala que el $67,9 \%$ de los usuarios de internet accedió a través del televisor durante el último mes y el 55,7\% en la última semana y, en total, el 27,1\% de los usuarios de internet reconoce que accede a la Red a través del televisor, situándose como el cuarto dispositivo y preferencia de acceso, detrás del teléfono móvil, ordenador y tablet, que ocupan el primer, segundo y tercer puesto, respectivamente.

Igualmente, el acceso de los navegantes a través de una smart TV experimentó un fuerte incremento, situándose en el $52,5 \%$ de los usuarios y la multitarea o utilización compartida de internet y TV es del 76\% (40,5\% frecuentemente y $35,6 \%$ de forma ocasional). Por lo que respecta al consumo de redes sociales, el $80,9 \%$ de los internautas españoles accedió a Facebook durante el último mes, seguido del 51,2\% a Instagram y el 40,9\% a Twitter.

Por otra parte, el EGM de Audiencia de internet (2019) (figura 1), informa que un 58\% de los usuarios accedió en algún momento a internet desde su televisor, con un incremento progresivo del consumo a través de dispositivos de acceso del tipo smartTV muy próximo al de las tablets, además de reseñar cuáles fueron las redes sociales con más usuarios: YouTube, en primer lugar, con un registro de 26,2 millones de usuarios, seguido de Facebook, con 19,4 millones. Twitter está en cuarto lugar, con 4,9 millones de usuarios visitantes únicos durante los últimos 30 días y, por lo que respecta a los sitios web de los canales de televisión, Antena3.com y RTVE.es ocupan el 9o y 10으 puesto, con 2,2 millones de usuarios cada una, seguidos de Lasexta.com, con 1,7 millones de usuarios, aunque si nos limitamos a las audiencias comprendidas entre los 14 y los 25 años, las horquillas son más favorables para el caso de internet con respecto a la televisión.

En consecuencia, los datos de audiencias confirman una tendencia y evolución, cada vez mayor, hacia la utilización de las denominadas segundas pantallas (Horning, 2017; Gil-de-Zúñiga; García-Perdomo; McGregor, 2015), que combina el visionado de televisión con otras pantallas conectadas a internet (teléfono, tablet, ordenador), como el mencionado proceso de consumo híbrido y de convergencia multimedia (Jenkins, 2008).

De hecho, la incorporación de las nuevas tecnologías digitales en los hábitos televisivos de la audiencia supuso que la centralidad de la pantalla de televisión fuera sustituida por una experiencia televisiva multipantalla (González-Neira; Quintas-Froufe, 2015).

Aunque no existe un consenso sobre el término y fenómeno segunda pantalla (Gil-de-Zúñiga; Liu, 2017; Horning, 2017; Gil-de-Zúñiga; García-Perdomo; McGregor, 2015; Giglietto; Selva, 2014) o doble pantalla (Shah et al., 2016; Vaccari; Chadwick; O’Loughlin, 2015), multipantalla (Dias, 2016) o uso de medios simultáneo complementario (Nee; Dozier, 2017), la teoría de las segundas -o múltiples pantallas- analiza los factores que predicen la selección y visionado de información, a través del empleo, único o combinado, de distintos dispositivos, involucrando la integración entre los medios de transmisión en vivo y las redes sociales (Vaccari; Chadwick; O'Loughlin, 2015), y, en definitiva, entre los medios tradicionales y los nuevos medios (Rúas-Araújo; Mazaira-Castro; Rodríguez-Vázquez, 2018).

Hay un creciente cuerpo de investigación que analiza los efectos de la multitarea en los medios (por ejemplo Kazakova et al., 2015) y, específicamente, de una segunda evaluación (Lin; Chiang, 2017) y las implicaciones que esta acción puede tener a la hora de aumentar la participación política de las personas -sobre todo en las noticias de contenido político y los debates electorales (Lin; Chiang, 2017; McGregor; Mourão, 2017)- y de lograr un mayor o menor recuerdo y comprensión, tal y como señalan Gottfried et al. (2017). Lo que hace que la investigación comparativa sea especialmente fructífera en este sentido es su reconocimiento de la relevancia de las condiciones contextuales y el intento de vincular variables de nivel macro y micro, que incluyen indicadores económicos, políticos, culturales e incluso de personalidad (Huber; Gil-de-Zúñiga; Liu, 2020), para explicar las diferencias entre países.

Se trata, en cualquier caso, de una audiencia consumidora y generadora de contenidos -prosumers-, que se convierte a su vez en prescriptora de información hacia otros usuarios que participan en las redes de forma pasiva (García-Galera; Valdivia, 2014).
Los datos de audiencias confirman una tendencia cada vez mayor, hacia la utilización de las segundas pantallas 
La web 2.0 ha promovido la instauración de un modelo de comunicación horizontal en el que se establece un comportamiento distinto al de las audiencias tradicionales: emisor y receptor son un mismo protagonista, su rol se fusiona quebrando el modelo rígido de los medios de comunicación tradicionales que prestaban menor atención a su público objetivo (Vicente-Torrico, 2019), un cambio que se convierte en oportunidad de comunicación, tanto para medios como para partidos políticos.

Este modelo da lugar a una nueva forma de entender la comunicación (Aparici; García-Marín, 2018), donde la audiencia social otorga valor a los contenidos en función de cómo afecta a su vida y, en consecuencia, dependerá del valor que le otorgue el público como prosumidor. En este sentido, destaca la transformación de esta audiencia, que funcionaba como actor pasivo receptor de mensajes sobre política (la llamada democracia representativa), a una democracia participativa-deliberativa, que facilita su propio contenido político, apropiándose a veces de la información o materiales que los mismos medios tradicionales generan.

En la actualidad, atender a lo que dice esta audiencia social está contemplado en las estrategias de comunicación de las campañas electorales, hasta el punto de que para establecer una comunicación exitosa es necesario atender a múltiples emisores que comparten y comen$\tan$ (Larrondo-Ureta, 2016). Un nuevo escenario en el que también es necesario tener en cuenta factores como la tendencia al infoentretenimiento en las publicaciones, sean de políticos, ciudadanos o medios de comunicación (Jivkova-Semova; Requeijo-Rey; Padilla-Castillo, 2017) e, incluso, ante la red social en la que se posicionan.

Plataformas como Instagram o YouTube, por ejemplo, han contribuido al desarrollo del perfil más humano y cercano de personajes políticos como parte de su campaña electoral, buscando la reacción de los usuarios y, en definitiva, moviendo hacia el voto a través de la personalización. Estas redes -más visuales- generan el comentario del lado más amable por parte de los internautas, algo que es aprovechado en época de campaña a través de la recreación de la vida del candidato, con el fin de aumentar su simpatía y crear valores personales de marca de los que hable esta audiencia social (Maarek, 2014).

Estamos por tanto ante una audiencia que busca el entretenimiento por encima de la información pura, también en política. Así, al igual que se muestra el lado humano del candidato en las redes sociales, en la selección de contenidos por parte de los internautas también prima la personalización política (Berrocal-Gonzalo; Campos-Domínguez; Redondo-García, 2012). Los usuarios prefieren los contenidos que giran alrededor del propio líder político más que al partido que representan, siendo los de tono humorístico los más atractivos, por encima de temas más trascendentales de la campaña electoral.

Un hecho que también se refleja en la red social Twitter, donde queda patente la proliferación de memes, por ejemplo, durante los debates de las elecciones generales de 2019, que inundaron esta red social y se convirtieron en lo más comentado y compartido, dejando en un segundo plano los temas tratados o ausentes en dichos debates. Claes y Deltell (2015) siguen una línea similar y establecen que la audiencia social únicamente comenta o menciona datos de actualidad política si estos son presentados dentro de espacios de entretenimiento.

Twitter es uno de los canales que se ha incorporado a los debates electorales, demostrando ser fundamental en la comunicación política actual durante la campaña electoral (Rodríguez-Fernández; Saavedra-Llamas, 2017; Jungherr, 2016; Lago-Vázquez et al., 2016; López-García, 2016). Una de las causas del predominio de uso de Twitter para difundir las propuestas políticas, como señalan López-Meri, Marcos-García y Casero-Ripollés (2017), es poder dirigirse directamente a la ciudadanía, sin la intermediación de los periodistas y los medios convencionales.

Varios autores (Ruiz-del-Olmo; Bustos-Díaz, 2017; Gamir-Ríos, 2016) apuntan a que la incorporación de Twitter se llevó a cabo en 2011, pero hubo que esperar hasta 2015 para su consolidación. Fue ese año cuando los debates electorales se convirtieron en los eventos con mayor audiencia social. Concretamente el primer debate electoral fue la emisión con más audiencia social de 2015 (2.491.539 tweets emitidos por 304.733 usuarios únicos), mientras que el segundo debate fue la tercera emisión con mayor audiencia social en España (Kantar Media, 2016).

En el debate sobre la utilización de las redes sociales, la cuestión de fondo es si éstas contribuyen a "ensanchar" el espacio público de la comunicación política, dando más protagonismo a los ciudadanos (Wolton, 2007) o, por el contrario, actúan como caja de resonancia de los medios tradicionales (López-Meri, 2017) y escaparate individual de los políticos, que utilizan esa red como extensión de las tradicionales estrategias de repetición de mensajes clave para su electorado potencial (Gelado-Marcos; Puebla-Martínez; Rubira-García, 2019), consolidando la idea de que los políticos están más interesados en la difusión de la información y en los retweets que en el debate (Campos-Domínguez, 2017).
La cuestión de fondo es si las redes sociales contribuyen a "ensanchar" el espacio público de la comunicación política o sólo actúan como caja de resonancia de los medios tradicionales 
En este sentido, Rodríguez-Fernández y Saavedra-Llamas (2017) consideran que la audiencia sí participa significativamente durante los debates a través de las redes, incentivada mayoritariamente por organizaciones, aunque los propios partidos no presenten una estrategia que dinamice la participación entre estas audiencias, y, por lo tanto, no exista integración real en la estrategia de las formaciones políticas. Por otro lado, en estos espacios destinados a campaña política, la participación de los públicos más jóvenes es escasa, millennials que frecuentan las redes sociales y las emplean más como fuentes de información y no como espacios de interacción (Lago-Vázquez et al., 2016).

En Twitter y Facebook los internautas manifiestan un comportamiento más participativo que de reacción (me gusta, me encanta, me asombra, me divierte, me entristece, me enfada) durante las campañas electorales (sobre todo Twitter, cuyo poder de convocatoria es más elevado en época de campaña electoral que sus competidoras).

Los públicos se informan de asuntos de índole social que copan los programas de partidos políticos y que los medios de comunicación tradicionales retransmiten, expresando su apoyo o malestar ante ellos. Muchos de estos temas se posicionan en primera línea de atención debido al uso de hashtags que estimulan la participación y permiten a su vez que otros usuarios entren en contacto con un círculo más allá del cercano y línea temporal convirtiendo la búsqueda de contenidos en funcional (Jungherr, 2015). Otras acciones específicas que permiten fomentar esta participación son a través de links que remiten a foros u otras webs relacionadas o material no retransmitido (Claes; Deltell, 2015).

Esta audiencia social no se impone bajo el dominio de los mensajes que se difunden a través de las redes sociales durante las campañas electorales. Es un público que registra información y tiene conciencia de ser audiencia, pero a su vez se manifiesta como un público que se resiste a otorgar la máxima credibilidad a los mensajes expuestos en cada plataforma y, sobre todo, con el poder de reaccionar ante ellos (Callejo-Gallejo; Agudo-Arroyo, 2019).
Las televisiones no están aprovechando las propias extensiones de sus canales a través de las redes sociales para generar conversación con los usuarios

De momento, lo cierto es que las televisiones no están aprovechando, siquiera, las propias extensiones de sus canales a través de las redes sociales para generar conversación con los usuarios de las mismas ni tampoco siguen una estrategia de comunicación clara y definida (Fontenla-Pedreira, 2018), al no cumplir con los principios dialógicos básicos (Kent; Taylor, 1998), fundamentalmente, en lo referido a la oportunidad de los usuarios de plantear preguntas y de generar conversación de los medios de comunicación con sus audiencias.

Además, es necesario incorporar nuevas métricas que permitan tener en cuenta a los nuevos espectadores que ven la televisión en otros dispositivos distintos del televisor. La información proporcionada por los audímetros de Kantar Media, operador único que en España mide las audiencias de televisión, se ha renovado. Desde principios del 2017 incluye en sus reportes también los datos de audiencia de invitados y desde principios de este año la medición de las segundas residencias. Asímismo ha llegado a un acuerdo con el operador único que mide la audiencia de los sites de internet en España, ComScore para desarrollar la denominada medición cross-media (Rodríguez-Vázquez; Direito-Rebollal, Silva-Rodríguez, 2018; Quintas-Froufe; González-Neira, 2016). Se trata de combinar la medición televisiva con una medición online multiplataforma (Santiago, 2017), para brindar una visión sin duplicidades del consumo online y de televisión.

La medición cross-media abarca el contenido de vídeo y texto, al que se puede acceder mediante un navegador, una aplicación, un smartphone, una tablet, un ordenador, un dispositivo de transmisión libre o un televisor. Este sistema híbrido ya está funcionando en los Países Bajos, donde se ofrece una visión completa de lo que ve la audiencia. Con esta medición se logrará conocer el número total de personas que consume un determinado contenido ya sea a través de la televisión o de plataformas online. Las novedades seguirán en el futuro: Nielsen está haciendo pruebas en EUA para medir las audiencias de la oferta de las plataformas OTT (over-the-top, de libre transmisión), capturando el

Es necesario incorporar nuevas métricas que permitan tener en cuenta a las nuevas audiencias que ven la televisión en dispositivos distintos del televisor

audio de sus emisiones. Y por supuesto ello ha conllevado la generación de una miríada de datos, que los operadores del sector tratan con técnicas de big data para convertirlos en conocimiento útil para sus decisiones de marketing y comunicación.

Con todo, queda para discutir la cuestión, ya indicada al comienzo, de si el debate político en las redes sociales contribuye a "ensanchar" el espacio público (Wolton, 2007), o por el contrario crea falsas ideas de consenso, a través de burbujas de opinión y cámaras de resonancia (echo-chambers), donde la información disidente se aísla o ignora.

Una respuesta y defensa ante la amenaza de la propaganda computacional, para

“manejar y distribuir información sesgada en las redes sociales de manera intencional” (Woolley; Howard, 2019), con la intención de

"sesgar, guiar, provocar, controlar, manipular o constreñir el comportamiento humano" (Danaher et al., 2017), lo cual ha llevado a hablar de democracia algorítmica o "algocracia". 
Finalmente, también es importante conocer en qué medida tanto los ciudadanos como los usuarios de las redes sociales dan relevancia (salience) a temas que los políticos no consideran centrales (López-López; Puentes-Rivera; Rúas-Araújo, 2017) o presentan agendas (McCombs; Shaw, 1974) y/o encuadres diferentes (Shaw, 1979). Sobre todo, teniendo en cuenta el evidente alineamiento y ensamblaje existente entre los temas fijados por la prensa durante la precampaña electoral y los contenidos de los candidatos durante la celebración de un debate televisado, cuyas intervenciones reproducen, a menudo, titulares y noticias de fuentes publicadas en los periódicos (Rúas-Araújo; Mazaira-Castro, 2019).

En definitiva, saber si el interés y opinión de los usuarios de las redes sociales coincide o no, en mayor o menor medida, con los intereses de los políticos y medios tradicionales, a la hora de enfrentarse a una campaña o debate electoral.

\section{Referencias}

AIMC (2020). Marco general de los medios de España.

https://www.aimc.es/a1mc-cOnt3nt/uploads/2020/01/marco2020.pdf

AIMC (2019). Infografía resumen 21ㅇ Navegantes en la Red.

https://www.aimc.es/otros-estudios-trabajos/navegantes-la-red/infografia-resumen-21o-navegantes-la-red/

Anstead, Nick (2016). "A different beast? Televised election debates in parliamentary democracies". The international journal of press/politics, v. 21, n. 4, pp. 508-526.

http://doi.org/10.1177/1940161216649953

Aparici, Roberto; García-Marín, David (2018). "Prosumidores y emirecs: análisis de dos teorías enfrentadas". Comunicar, v. 26, n. 55, pp. 71-79.

https://doi.org/10.3916/C55-2018-07

Barlovento Comunicación (2019). Los debates electorales en televisión.

https://www.barloventocomunicacion.es/informes-barlovento/audiencias-debates-electorales

Barrientos, Almudena; Caldevilla-Domínguez, David; Vargas-Delgado, José-Jesús (2019). “El protocolo, la puesta en escena y la persuasión en los debates políticos televisados". Redmarka. Revista de marketing aplicado, v. 23, n. 4, pp. 17-27.

https://doi.org/10.17979/redma.2019.23.3.5872

Benoit, William L.; Hansen, Glenn J.; Verser, Rebecca M. (2003). "A meta-analysis of the effects of viewing U.S. presidential debates". Communication monographs, v. 70, n. 4, pp. 335-350.

https://doi.org/10.1080/0363775032000179133

Berrocal-Gonzalo, Salomé (2005). “La información política en televisión: ¿apatía o interés entre los telespectadores?”. Comunicar, v. 25, n. 5.

https://doi.org/10.3916/C25-2005-047

Berrocal-Gonzalo, Salomé; Campos-Domínguez, Eva; Redondo-García, Marta (2012). “Comunicación política en internet: la tendencia al 'infoentretenimiento' político en YouTube". Estudios sobre el mensaje periodístico, v. 18, n. 2, pp. 643-659.

https://doi.org/10.5209/rev_ESMP.2012.v18.n2.41037

Blais, André; Perrella, Andrea (2008). "Systemic effects of televised candidates' debates". The international journal of press/politics, v. 13, n. 4, pp. 451-464.

http://doi.org/10.1177/1940161208323548

Bruun, Hanne (2016). "The prism of change: 'continuity' in public service television in the digital era". Nordicom review, v. 37, n. 2, pp. 33-49.

https://doi.org/10.1515/nor-2016-0016

Callejo-Gallego, Javier (1995). La audiencia activa: el consumo televisivo, discursos y estrategias. Centro de Investigaciones Sociológicas: Madrid. ISBN: 9788474762211

Callejo-Gallego, Javier; Agudo-Arroyo, Yolanda (2019). “Análisis cuantitativo y cualitativo de la audiencia de las cuentas del Estado español en Facebook y Twitter". El profesional de la información, v. 28, n. 5, pp. 1215-1224.

https://doi.org/10.3145/epi.2019.sep.19

Campos-Domínguez, Eva (2017). “Twitter y la comunicación política”. El profesional de la información, v. 26, n. 5, pp. 785-793.

https://doi.org/10.3145/epi.2017.sep.01 
Cantavella-Blasco, Juan; Bullough, Rachel; Curiel-Calleja, Luis-Antonio; Morales-Blanco-Sieger, Begoña; Mejía-Chiang, César; Pittaro, Esteban (2008). "Algunos aspectos lingüísticos de los debates electorales Zapatero-Rajoy 2008". Estudios sobre el mensaje periodístico, v. 14, pp. 79-98.

https://revistas.ucm.es/index.php/ESMP/article/view/ESMP0808110079A

Carpentier, Nico (2001). "Managing audience participation: The construction of participation in an audience discussion programme". European journal of communication, v. 16, n. 2, pp. 209-232.

http://doi.org/10.1177/0267323101016002004

Casero-Ripollés, Andreu (2017). "Producing political content for web 2.0: Empowering citizens and vulnerable populations". El profesional de la información, v. 26, n. 1, pp. 13-19.

https://doi.org/10.3145/epi.2017.ene.02

Chadwick, Andrew (2013). The hybrid media system: Politics and power. Oxford: Oxford University Press. ISBN: 9780 199759484

Chadwick, Andrew; O'Loughlin, Ben; Vaccari, Cristian (2017). "Why people dual screen political debates and why it matters for democratic engagement". Journal of broadcasting \& electronic media, v. 61, n. 2, pp. 220-239.

https://doi.org/10.1080/08838151.2017.1309415

Chaffee, Steven H. (1978). "Presidential debates - are they helpful to voters?”. Communication monographs, v. 45, n. 4, pp. 330-346.

https://doi.org/10.1080/03637757809375978

Claes, Florencia; Deltell, Luis (2015). "Audiencia social en Twitter: hacia un nuevo modelo de consumo televisivo". Trípodos, v. 36, pp. 111-132.

https://www.raco.cat/index.php/Tripodos/article/view/301862/391480

Danaher, John; Hogan, Michael J.; Noone, Chris; Kennedy, Rónán; Behan, Anthony; De-Paor, Aisling; Felzmann, Heike; Haklay, Muki; Khoo, Su-Ming; Morison, John; Murphy, Maria-Helen; O’Brolchain, Niall; Schafer, Burkhard; Shankar, Kalpana (2017). "Algorithmic governance: developing a research agenda through the power of collective intelligence". Big data \& society, v. 4, n. 2, pp. 1-21.

https://doi.org/10.1177/2053951717726554

Deller, Ruth (2011). "Twittering on: Audience research and participation using Twitter". Participations, v. 8, n. 1, pp. 216-245.

https://www.participations.org/Volume\%208/Issue\%201/deller.htm

D’heer, Evelien; Verdegem, Pieter (2015). "What social media data mean for audience studies: A multidimensional investigation of Twitter use during a current affairs TV programme". Information, communication \& society, v. 18, n. 2 , pp. 221-234.

https://doi.org/10.1080/1369118X.2014.952318

Dias, Patricia (2016). "Motivations for multi-screening: An exploratory study on motivations and gratifications". European journal of communication, v. 31, n. 6, pp. 678-693.

https://doi.org/10.1177/0267323116674111

Echegaray-Eizaguirre, Lázaro; Peñafiel-Saiz, Carmen (2013). "La utilización de las redes sociales como nuevas herramientas aplicadas al análisis de audiencia”. Trípodos, v. 33, pp. 157-172.

https://core.ac.uk/download/pdf/39028837.pdf

EGM (2019). Audiencia de internet.

https://www.aimc.es/a1mc-cOnt3nt/uploads/2019/04/internet119.pdf

Elmer, Greg (2013). "Live research: Twittering an election debate”. New media \& society, n. 15, v. 1, pp. $18-30$. https://doi.org/10.1177/1461444812457328

Fontenla-Pedreira, Julia (2018). “Las televisiones autonómicas en las redes sociales”. En: Conde, Erika; Fontenla, Julia; Rúas, José. Debates electorales televisados: del antes al después, pp. 235-258. La Laguna (Tenerife): Cuadernos artesanos de comunicación, ISBN: 9788417314149

Gallego-Reguera, María; Bernárdez-Rodal, Asunción (2017). “Influencia y repercusión mediática de los debates 'cara a cara' celebrados ante las elecciones generales de 2008 en España: José Luis Rodríguez Zapatero (PSOE) vs Mariano Rajoy (PP)". Vivat academia, n. 141, pp. 139-154.

http://doi.org/10.15178/va.2017.141.139-154

Gamir-Ríos, José (2016). “Blogs, Facebook y Twitter en las elecciones generales de 2011. Estudio cuantitativo del uso de la web 2.0 por parte de los cabezas de lista del PP y del PSOE”. Dígitos. Revista de comunicación digital, v. 2, pp. 101-120. https://revistadigitos.com/index.php/digitos/article/view/53 
García-Galera, Mari-Carmen; Valdivia, Angharad (2014). “Media prosumers, participatory culture of audiences and media responsability". Comunicar, v. 22, n. 43, pp. 10-13.

https://doi.org/10.3916/C43-2014-a2

García-Martín, Javier (2015). “La cobertura mediática de los debates electorales en España”. Revista española de ciencia política, n. 38, pp. 135-161.

https://recyt.fecyt.es/index.php/recp/article/view/37666

Gelado-Marcos, Roberto; Puebla-Martínez, Belén; Rubira-García, Rainer (2019). "Comunicación política y personalización de contenidos: ¿es Twitter el nuevo medio de referencia para la interacción entre gobernantes y gobernados?". AdResearch ESIC, v. 20, n. 20, pp. 8-19.

https://doi.org/10.7263/adresic-020-01

Giglietto, Fabio; Selva, Donatella (2014). "Second screen and participation: A content analysis on a full season dataset of tweets". Journal of communication, v. 64, n. 2, pp. 260-277.

https://doi.org/10.1111/jcom.12085

Gil-de-Zúñiga, Homero; García-Perdomo, Víctor; McGregor, Shannon (2015). "What is second screening? Exploring motivations of second screen use and its effect on online political participation". Journal of communication, v. 65, n. 5, pp. $793-815$. https://doi.org/10.1111/jcom.12174

Gil-de-Zúñiga, Homero; Liu, James (2017). "Second screening politics in the social media sphere: Advancing research on dual screen use in political communication with evidence from 20 countries". Journal of broadcasting \& electronic media, v. 61, pp. 193-219.

https://doi.org/10.1080/08838151.2017.1309420

González-López, Felipe (2019). “Big data, algoritmos y política: las ciencias sociales en la era de las redes digitales". Cinta de Moebio. Revista de epistemología de ciencias sociales, n. 65, pp. 267-280.

https://doi.org/10.4067/S0717-554X2019000200267

González-Neira, Ana; Quintas-Froufe, Natalia (2015). “La revisión del concepto de televisión social y sus audiencias”. En: Quintas-Froufe, Natalia; González-Neira, Ana. La participación de la audiencia en la televisión: de la audiencia activa a la social, pp. 13-26. Madrid: AIMC. ISBN: 9788460842422

Gottfried, Jeffrey A.; Hardy, Bruce W.; Holbert, R. Lance; Winneg, Kenneth M.; Jamieson, Kathleen-Hall (2017). "The changing nature of political debate consumption: Social media, multitasking, and knowledge acquisition". Political communication, v. 34, pp. 172-199.

https://doi.org/10.1080/10584609.2016.1154120

Greenwood, Shannon; Perrin, Andrew; Duggan, Maeve (2016). Social media update 2016. Pew Research Center. https://www.pewresearch.org/internet/wp-content/uploads/sites/9/2016/11/PI_2016.11.11_Social-Media-Update_FINAL.pdf

Hallin, Daniel C.; Mancini, Paolo (2004). Comparing media systems. Three models of media and politics. Cambridge University Press. ISBN: 9780521543088

Horning, Michael A. (2017). "Interacting with news: Exploring the effects of modality and perceived responsiveness and control on news source credibility and enjoyment among second screen viewers". Computers in human behavior, v. 73, pp. 273-283.

https://doi.org/10.1016/j.chb.2017.03.023

Horowitz, Minna-Aslama (2015). "Public service media and challegue of crossing borders: Assessing new models". Medijske studije, v. 6, n. 12, pp. 80-90.

https://hrcak.srce.hr/ojs/index.php/medijske-studije/article/view/6155

Huber, Brigitte; Gil-de-Zúñiga, Homero; Liu, James (2020). “Assessing political second screening behavior and personality traits: The roles of economic development, freedom of expression and monochromatic vs. polychromatic cultures". Telematics and informatics.

https://doi.org/10.1016/j.tele.2020.101365

Huertas-Bailén, Amparo (2002). La audiencia investigada. Barcelona: Editorial Gedisa. ISBN: 9788474329575

Humanes, María-Luisa (2014). “Exposición selectiva y partidismo de las audiencias en España. El consumo de información política durante las campañas electorales de 2008 y 2011”. Palabra clave, v. 17, n. 3, pp. 773-802.

https://doi.org/10.5294/pacla.2014.17.3.9

IAB (2019). Estudio anual de redes sociales 2019.

https://iabspain.es/estudio/estudio-anual-de-redes-sociales-2019

Jenkins, Henry (2008). Convergence culture: La cultura de la convergencia de los medios de comunicación. Barcelona: Paidós. ISBN: 9780814742952 
Jivkova-Semova, Dimitrina; Requeijo-Rey, Paula; Padilla-Castillo, Graciela (2017). "Usos y tendencias de Twitter en la campaña a elecciones generales españolas del 20d de 2015: hashtags que fueron trending topic". El profesional de la información, v. 26, n. 5, pp. 824-837.

https://doi.org/10.3145/epi.2017.sep.05

Jungherr, Andreas (2015). Analyzing political communication with digital trace data. Switzerland: Springer. ISBN: 9783 319203188

Jungherr, Andreas (2016). "Twitter use in election campaigns: A systematic literature review". Journal of information technology and politics, v. 13, n. 1, pp. 72-91.

https://doi.org/10.1080/19331681.2015.1132401

Kantar Media (2016). Kantar Twitter TV ratings.

https://www.kantarmedia.com

Kazakova, Snezhanka; Cauberghe, Veroline; Pandelaere, Mario; De-Pelsmacker, Patrick (2015). "Can't see the forest for the trees? The effect of media miltitasking on cognitive processing style". Media psychology, v. 18, n. 4, pp. 425-450. https://doi.org/10.1080/15213269.2015.1006789

Kent, Michael L.; Taylor, Maureen (1998). "Building dialogic relationships through the word wide web". Public relations review, v. 24, n. 3, pp. 321-334.

https://doi.org/10.1016/S0363-8111(99)80143-X

Lago-Vázquez, Diana; Direito-Rebollal, Sabela; Rodríguez-Vázquez, Ana-Isabel; López-García, Xosé (2016). “El consumo millennial de información política en televisión y redes sociales. Análisis de la campaña Elecciones Generales en España 2015". Revista latina de comunicación social, n. 71, pp. 1.151-1.169.

https://doi.org/10.4185/RLCS-2016-1139

Larrondo-Ureta, Ainara (2016). “Comunicación organizacional ante el reto de la estrategia multiplataforma y 2.0: la experiencia de los partidos políticos en el País Vasco". El profesional de la información, v. 25, n. 1, pp. 114-123.

https://doi.org/10.3145/epi.2016.ene.11

Lin, Trisha; Chiang, Yi-Hsuan (2017). "Dual screening: Examining social predictors and impact on online and offline political participation among Taiwanese internet users. Journal of broadcasting \& electronic media, n. 61, pp. $240-263$. https://doi.org/10.1080/08838151.2017.1309419

Livingstone, Sonia (2013). "The participation paradigm in audience research". The communication review, v. 16, pp. 21-30. https://doi.org/10.1080/10714421.2013.757174

López-García, Guillermo (2016). “'New' vs 'old' leaderships: the campaign of Spanish general elections 2015 on Twitter”. Communication \& society, v. 29, n 3, pp. 149-167.

https://doi.org/10.15581/003.29.3.149-168

López-García, Guillermo; Llorca-Abad, Germán; Valera-Ordaz, Lidia; Peris-Blanes, Àlvar (2018). "Los debates electorales, ¿̇el último reducto frente la mediatización? Un estudio de caso de las elecciones generales españolas de 2015". Palabra clave, v. 21, n. 3, pp. 772-797.

https://doi.org/10.5294/pacla.2018.21.3.6

López-López, Paulo-Carlos; Oñate, Pablo (2019). “De la videopolítica a la ciberpolítica: debate entre candidatos y televisiones en cinco elecciones presidenciales”. El profesional de la información, v. 28, n. 5, e280512.

https://doi.org/10.3145/epi.2019.sep.12

López-López, Paulo-Carlos; Puentes-Rivera, Iván; Rúas-Araújo, José (2017). “La comunicación política en las elecciones gallegas del 25 de septiembre de 2016: la agenda temática en el debate de la TVG". En: Congreso Latina de comunicación social. Del verbo al bit, pp.1431-1460.

https://doi.org/10.4185/cac116edicion2

López-Meri, Amparo (2017). “Contribución ciudadana al debate electoral y su cobertura periodística en Twitter". Prisma social, v. 18, pp. 1-33.

https://revistaprismasocial.es/article/view/1466

López-Meri, Amparo; Marcos-García, Silvia; Casero-Ripollés, Andreu (2017). "What do politicians do on Twitter? Functions and communication strategies in the Spanish electoral campaign of 2016". El profesional de la información, v. 26, n. 5, pp. 795-804.

https://doi.org/10.3145/epi.2017.sep.02

López-Rico, Carmen-María; Peris-Blanes, Àlvar (2017). “Agenda e imagen de los candidatos de las elecciones generales de 2015 en España en programas televisivos de infoentretenimiento". El profesional de la información, v. 26, n. 4, pp. 611-620. https://doi.org/10.3145/epi.2017.jul.05 
Lull, James (1997). Medios, comunicación, cultura. Buenos Aires: Amorrortu. ISBN: 9789505186600

Maarek, Philippe J. (2014). "Politics 2.0: New forms of digital political marketing and political communication". Trípodos, n. 34, pp. 13-22.

http://www.tripodos.com/index.php/Facultat_Comunicacio_Blanquerna/article/view/163

Martín-Jiménez, Virginia; Berrocal-Gonzalo, Salomé (2017). “Innovación y consolidación del 'infoentretenimiento’ político: una perspectiva histórica”. Historia y Comunicación social, v. 22, n. 1, pp. 207-219.

https://doi.org/10.5209/HICS.55908

Martínez-Pandiani, Gustavo (2006). “El impacto de la televisión en la comunicación política moderna”. Signos universitarios, v. 25, n. 1, pp. 69-88.

https://p3.usal.edu.ar/index.php/signos/article/download/2955/3582

Marzal-Felici, Javier; Zallo-Elguezabal, Ramón (2016). “Presentación: Las televisiones públicas de proximidad ante los retos de la sociedad digital”. Communication \& society, v. 29, n. 4, pp. 1-7.

https://revistas.unav.edu/index.php/communication-and-society/article/view/35802

McCombs, Maxwell E.; Shaw, Donald L. (1972). "The agenda-setting function of mass media”. The public opinion quarterly, v. 36, n. 2, pp. 176-187.

https://www.jstor.org/stable/2747787

McGregor, Shannon C.; Mourão, Rachel R. (2017). "Second screening Donald Trump: Conditional indirect effects on political participation. Journal of broadcasting \& electronic media, v. 61, n. 2. pp. 264-290.

https://doi.org/10.1080/08838151.2017.1309418

Montagut, Marta; Carrillo, Nereida (2017). “Estrategias de espectacularización en las tertulias políticas televisivas. Caso de la cobertura de las elecciones municipales de Barcelona de 2015". El profesional de la información, v. 26, n. 4, pp. 621-629.

https://doi.org/10.3145/epi.2017.jul.06

Morley, David (1996). Televisión, audiencias y estudios culturales. Buenos Aires: Amorrortu. ISBN: 9789505186440

Nee, Rebecca-Coates; Dozier, David M. (2017). "Second screen effects: Linking multiscreen media use to televisión engagement and incidental learning". Convergence: The international journal of research into new media technologies, $\mathrm{v}$. 23, n. 2, pp. 214-226.

https://doi.org/10.1177/1354856515592510

Pew Research Center (2019). Social media fact sheet.

https://www.pewresearch.org/internet/fact-sheet/social-media

Proaño, Luis-Eladio (2002). “Debate presidencial en la TV: ¿Ayuda a ganar una elección?”. Chasqui, n. 79, pp. 30-37. https://revistachasqui.org/index.php/chasqui/article/view/1444

Quintas-Froufe, Natalia; González-Neira, Ana (2016). “Consumo televisivo y su medición en España: camino hacia las audiencias híbridas". El profesional de la información, v. 25, n. 3, pp. 376-383.

https://doi.org/10.3145/epi.2016.may.07

Reguero-Sanz, Itziar; Martín-Jiménez, Virginia (2020). “Programas matinales televisivos: un análisis cuantitativo de las entrevistas a políticos en TVE y Antena 3". Palabra clave, v. 23, n.1, e2315.

https://doi.org/10.5294/pacla.2020.23.1.5

Reinemann, Carsten; Wilke, Jürgen (2007). "It's the debates, stupid! How the introduction of televised debates changed portrayal of chancellor candidates in the German press, 1949-2005". Harvard international journal of press/politics, v. 12, n. 4, pp. 92-111.

https://doi.org/10.1177/1081180X07307185

Rodríguez-Fernández, Leticia; Saavedra-Llamas, Marta (2017). “Debate electoral 13-J: la audiencia social en la estrategia digital de los partidos políticos". Trípodos, v. 41, pp. 173-191.

http://www.tripodos.com/index.php/Facultat_Comunicacio_Blanquerna/article/view/441/508

Rodríguez-Vázquez, Ana-Isabel; Direito-Rebollal, Sabela; Silva-Rodríguez, Alba (2018). “Audiencias crossmedia: Nuevas métricas y perfiles profesionales en los medios españoles". El profesional de la información, v. 27, n. 4, pp. $793-800$. https://doi.org/10.3145/epi.2018.jul.08

Rúas-Araújo, José; Mazaira-Castro, Andrés (2019). “Agenda mediática y política: ¿Amistados peligrosas? Un análisis desde el fact-checking". En: Conde, Erika; Fontenla, Julia; Rúas, José. Debates electorales televisados: del antes al después, pp. 151-172. La Laguna (Tenerife): Cuadernos artesanos de comunicación. ISBN: 9788417314149 
Rúas-Araújo, José; Mazaira-Castro, Andrés; Rodríguez-Vázquez, Ana-Isabel (2018). “Nuevos medios y medios tradicionales en la red. Espacios de opinión e interacción política en la era Trump”. Icono14, v. 16, n. 1, pp. 86-113. https://icono14.net/ojs/index.php/icono14/article/view/1118 https://doi.org/10.7195/ri14.v16i1.1118

Ruiz-del-Olmo, Francisco-Javier; Bustos-Díaz, Javier (2017). "La evolución del debate televisivo como herramienta de comunicación política. Análisis del caso español: de la televisión a Twitter". Informação \& sociedade, v. 27, n. 2, pp. 235-252. https://periodicos.ufpb.br/ojs/index.php/ies/article/view/32491

Santiago, Fernando (2017). Fragmentación de la audiencia: retos de la medición. Barcelona: Asociación para la Investigación de Medios de Comunicación, AIMC. https://www.aimc.es/a1mc-c0nt3nt/uploads/2017/09/170609_fsc_jornada_occ_barcelona-2.pdf

Selva, Donatella (2016). "Social television: Audience and political engagement". Television and new media, v. 17, n. 2, pp. 159-173.

https://doi.org/10.1177/1527476415616192

Shah, Dhavan V.; Hanna, Alex; Bucy, Erik P.; Lassen, David S.; Van-Thomme, Jack; Bialik, Kristen; Yang, JungHwan; Pevehouse, Jon C. W. (2016). "Dual screening during presidential debates: Political nonverbals and the volume and valence of online expression". American behavioral scientist, v. 60, n. 14, pp. 1816-1843.

https://doi.org/10.1177/0002764216676245

Shaw, Eugene (1979). "Agenda-setting and mass communication theory". International communication gazette, v. 25, n. 2, pp. 96-105. https://doi.org/10.1177/001654927902500203

Téllez, Nilsa; Muñiz, Carlos; Ramírez, Jacobo (2010). “Función discursiva en los debates televisados. Un estudio transcultural de los debates políticos en México, España y Estados Unidos”. Palabra clave, v. 13, n. 2, pp. 251-270.

https://doi.org/10.5294/pacla.2010.13.2.2

Trappel, Josef (2016). "Taking the public service remit forward across the digital boundary". International journal of digital television, v. 7, n. 3, pp. 273-295.

https://doi.org/10.1386/jdtv.7.3.273_1

Tremblay, Gaëtan (2016). "Public service media in the age of digital networks". Canadian journal of communication, v. 41, n. 4, pp. 191-206.

https://doi.org/10.22230/cjc.2016v41n1a3062

Trilling, Damian (2015). "Two different debates? Investigating the relationship between a political debate on TV and simultaneous comments on Twitter". Social science computer review, v. 33, n. 3, pp. 259-276.

https://doi.org/10.1177/0894439314537886

Vaccari, Cristian; Chadwick, Andrew; O'Loughlin, Ben (2015). "Dual screening: The political media events, social media and citizen engagement". Journal of communication, v. 65, pp. 1041-1061.

https://doi.org/10.1111/jcom.12187

Vázquez-Barrio, Tamara; Cebrián-Guinovart, Elena (2018). "Telerrealidad y política se unen: Temario y estrategias discursivas de los políticos en Planeta Calleja y En la tuya o en la mía". Communication \& society, v. 31, n. 1, pp. 73-89. http://dadun.unav.edu/handle/10171/54620

Vergeer, Maurice; Franses, Philip-Hans (2016). "Live audience responses to live televised election debates: Time series analysis of issue salience and party salience on audience behavior". Information, communication \& society, v. 19, n. 10, pp. 1390-1410.

https://doi.org/10.1080/1369118X.2015.1093526

Vicente-Torrico, David (2019). “Nuevas herramientas, viejas costumbres. El contenido generado por los usuarios sobre el cambio climático en YouTube”. Ámbitos: revista internacional de comunicación, v. 46, pp. 28-47.

https://doi.org/10.12795/Ambitos.2019.i46.03

Webster, James; Phalen, Patricia F.; Lichty, Lawrence W. (2014). Rating analysis: Audience measurement and analytics. New York: Routledge. ISBN: 9780415526524

Woolley, Samuel C.; Howard, Philip N. (2019). Computational propaganda: political parties, politicians, and political manipulation on social media. Oxford: Oxford University Press. ISBN: 9780190931407

Wolton, Dominique (2007). Pensar la comunicación. Argentina: Prometeo. ISBN: 9789875741324

Yawn, Mike; Ellsworth, Kevin; Beatty, Bob; Kahn, Kim-Fridkin (1998). “How a presidential primary debate changed attitudes of audience members". Political behavior, v. 20, n. 2, pp. 155-181.

https://www.jstor.org/stable/586580 


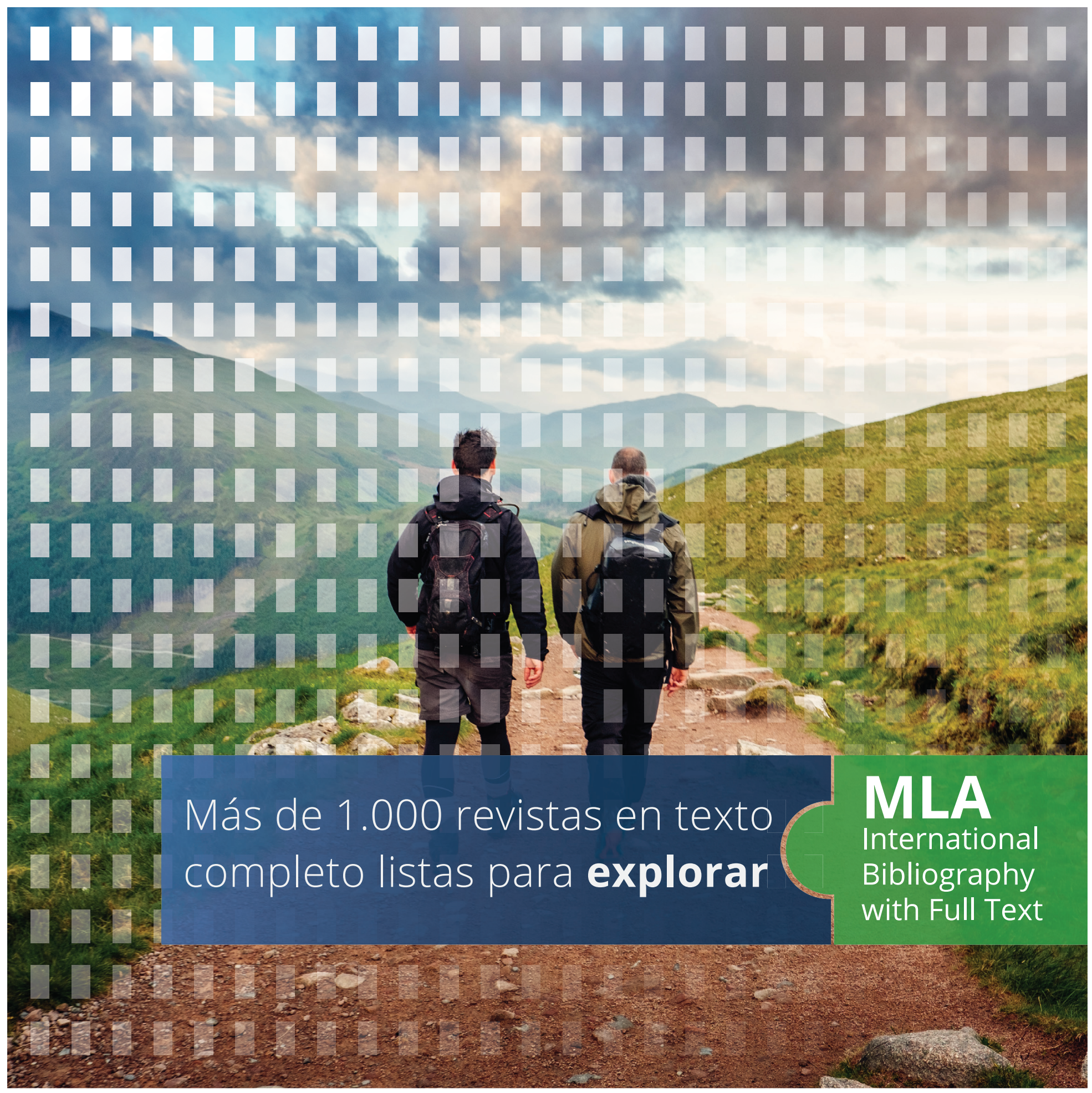

De la Modern Language Association (MLA) y EBSCO, esta nueva base de datos combina el índice definitivo para el estudio de la lengua, la literatura, la lingüística, la retórica y la composición, el folclore y el cine con texto completo de más de 1.000 revistas, entre las que se incluyen muchas de las revistas más utilizadas en MLA International Bibliography. Los buenos resultados están garantizados.

Solicite una prueba gratuita
Para programar una prueba gratuita, comuníquese con su representante de EBSCO o visítenos: exploremla.is/14 